\title{
Structural Characterization of Tethered HIV-1 VLPs by Light Microscopy and Cryo-Electron Tomography
}

\author{
Joshua D. Strauss, Jason E. Hammonds, Paul W. Spearman, and Elizabeth R. Wright
}

Division of Pediatric Infectious Diseases, Emory University School of Medicine, Children's Healthcare of Atlanta, Atlanta, GA 30322, USA

Tetherin (BST-2/CD317) is a host cell restriction factor that acts on wide range of enveloped viruses by physically linking virions to the host cell's plasma membrane [1]. Retention of virions at the plasma membrane requires dimerization of tetherin monomers, which is mediated by tetherin's large ectodomain and membrane insertions of tetherin's N-terminal single transmembrane domain and Cterminal glycophosphatidylinositol (GPI) anchor. Cells possessing tethered virions and virus-like particles (VLPs) show distinct phenotype consisting of large puncta of aggregated virions that cluster with each other and along the host cell's plasma membrane [2-5]. Imaging of tethered virions by thinsection transmission electron microscopy (TEM) and immuno-TEM has been limited to the examination of chemically fixed and heavy metal stained specimens. To characterize the native structure of tethered HIV-1 VLPs we have utilized a correlative microscopy approach by combining live cell imaging with cryo-electron tomography (cryo-ET) to directly visualize HIV-1 VLPs tethered to human cells.

Subconfluent cultures of HT1080 cells were grown on collagen coated gold Quantifoil R2/2 grids (Quantifoil). Cells were transfected with eGFP-tetherin and mCherry-Gag (1:3 ratio of labeled to unlabeled HIV-1 Gag) using Lipofectamine 2000 (Invitrogen) transfection reagent. Live cells were imaged 8 to 10 hours post-transfection with a DeltaVision imaging workstation using a $60 \mathrm{X}$ oil immersion objective lens as described previously [6]. Grids with intact, labeled cells (Fig 1a) were cryoimmobilized by rapid immersion into liquid ethane using the CP3 (Gatan). Cryo-specimens were examined with a JEOL JEM-2200FS FEG-TEM operated at $200 \mathrm{kV}$ with the in-column Omega energy filter operated with a slit width of $20 \mathrm{eV}$. Images were recorded on the $4 \mathrm{~K} \times 4 \mathrm{~K}$ Gatan Ultrascan CCD camera. Image montages were generated using SerialEM [7], as illustrated in Fig 1b, to identify areas containing tethered HIV-1 VLPs. Clusters of VLPs were seen in low-dose images along the cell periphery, which was similar to previously published TEM studies [2-5]. Single-axis tilt series were collected with SerialEM using angular range of $-62^{\circ}$ to $62^{\circ}$ with a $2^{\circ}$ increment. The total electron dose was limited to $\sim 120 \mathrm{e}^{-/} \AA^{2}$ The tilt series images were acquired with a defocus between 4-6 $\mu \mathrm{m}$ and a pixel size of $0.764 \mathrm{~nm}$. In the three-dimensional reconstructions, rod-shaped densities linking together tethered HIV-1 VLPs (Fig 1c) were observed. These filamentous structures were not resolved in previous TEM studies. This demonstrates the benefit of imaging whole cells and HIV-1 VLPs by cryoET in order to characterize the native structure of tetherin. Detailed structural analysis of the tomograms is currently underway and will provide insight into the spatial organization of the tetherin complexes that link the HIV-1 VLPs together and to the cell's plasma membrane. 


\section{References:}

[1] Neil, S.J. Microbiol Immunol. 37, (2013), p. 67

[2] Neil, S.J., T. Zang, and P.D. Bieniasz, T. Nature. 451, (2008), p. 425

[3] Fitzpatrick, K., et al., PLoS Pathog. 6, (2010), p. e1000701

[4] Hammonds, J., et al., PLoS Pathog. 6, (2010), p. e1000749

[5] Perez-Caballero, D., et al., Cell. 139, (2009), p. 499

[6] Chu, H., et al., Cell Host Microbe. 12, (2012), p. 360

[7] Mastronarde, D.N., J Struct Biol. 152, (2005), p. 36

[8] This research was supported by funds from Emory University, Children's Healthcare of Atlanta, the Emory Center for AIDS Research, the Georgia Research Alliance, and the National Science Foundation to E.R.W. Cryo-EM/ET data was collected at the Emory University Robert P. Apkarian Integrated Electron Microscopy Core.

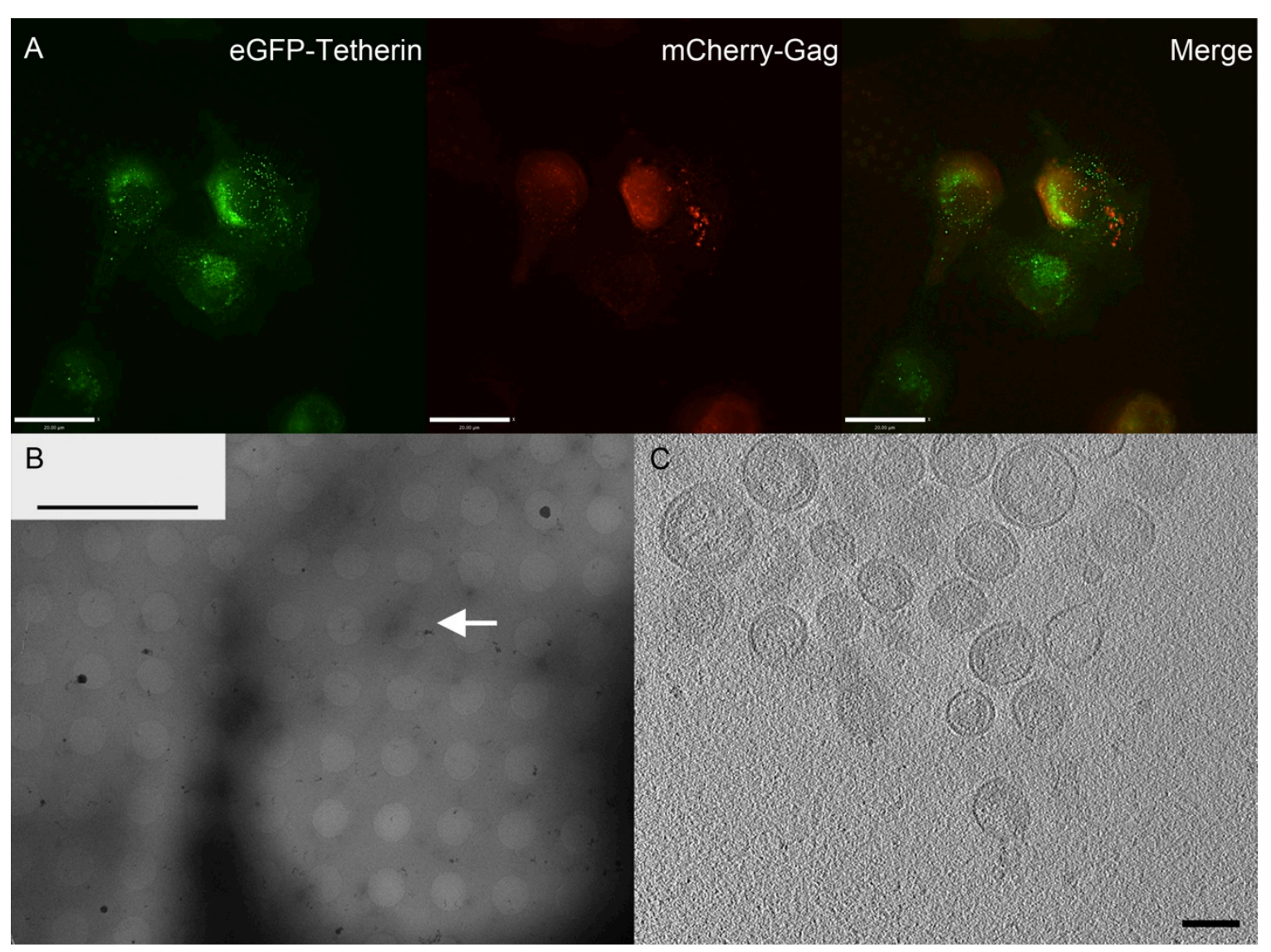

Figure 1. Examination of tethered HIV-1 VLPs by light and cryo-electron microscopy. (A) Cotransfected cells with puncta rich in eGFP-tetherin and mCherry-Gag along the periphery of the cell. (B) Cell extension with clusters of HIV-1 VLPs (white arrow) imaged by cryo-EM. (C) Slice through a 3D reconstruction of tethered HIV-1 VLPs from the same area in (B). Scale bars 20, 10 and $0.1 \mu \mathrm{m}$. 